\title{
A New Random Forest Method for One-Class Classification
}

\author{
Chesner Désir ${ }^{1}$, Simon Bernard $^{2}$, Caroline Petitjean $^{1}$, and Laurent Heutte ${ }^{1}$ \\ 1 Université de Rouen, LITIS EA 4108, BP 12, 76801 Saint-Etienne-du-Rouvray, France \\ Laurent. Heutte@univ-rouen. fr \\ 2 Université de Liège, Department of EECS et GIGA-Research, Grande Traverse, 10 - B-4000 \\ Liège - Belgium
}

\begin{abstract}
We propose a new one-class classification method, called One Class Random Forest, that is able to learn from one class of samples only. This method, based on a random forest algorithm and an original outlier generation procedure, makes use of the ensemble learning mechanisms offered by random forest algorithms to reduce both the number of artificial outliers to generate and the size of the feature space in which they are generated. We show that One Class Random Forests perform well on various UCI public datasets in comparison to few other state-of-the-art one class classification methods (gaussian density models, Parzen estimators, gaussian mixture models and one-class SVMs).
\end{abstract}

Keywords: One-class classification, decision trees, ensemble methods, random forests, outlier generation.

\section{Introduction}

One-class classification (OCC) is a binary classification task for which only one class of objects (the target class) is available for learning. OCC paradigm mainly deals with applications for which collecting counter-example samples (outliers) is impossible, like authorship verification, handwritten character or typist recognition [12], mobilemasquerader detection, machine or structure health monitoring [3], etc. As for traditional supervised learning, OCC literature usually opposes density-based methods to discriminative (or frontier-based) methods [4]. Density-based methods aim at estimating the probability density function of the target data and are thus straightforwardly applicable to OCC. The most used techniques among these methods are Parzen windowing and Mixtures of Gaussians (MoG) [5]. However, density-based methods are rarely effective for high dimensional data and usually require a large number of training samples to provide a reasonably good estimate of the distribution [5]. Discriminative approaches, based on the construction of a decision frontier between classes to discriminate, have also been introduced for OCC [2]. Their main difficulty is to synthesize the class of outliers in order to model the decision frontier. This is usually done by either using kernels, as in SVM-based methods [2], or by artificially generating outliers during training as in [1]. In this latter case, artificially generated outliers are often assumed to be uniformly distributed, so as to cover the whole domain of variation of the feature space. This implies to generate an exponential and thus expensive amount of outliers 
with respect to the dimension of the feature space, and as a consequence, this way of generating outliers is often inaccurate or unusable, especially with high dimensional data.

Ensemble methods are not so used to tackle OCC [4 5], though these methods are known to be powerful for traditional learning tasks [6]. As we will show, they offer some interesting randomization mechanisms that may be used to reduce both the number of outliers to generate and the dimension of the feature space in which outliers are generated. We investigate in this paper the use of ensembles of decision trees, such as random forests [7], that embed the interesting randomization mechanisms evoked above and that have proved their efficiency over single classifiers on various standard classification tasks [6]. We thus propose a new ensemble approach for OCC, called One-Class Random Forest (OCRF), based on a random forest algorithm and designed to tackle issues relative to the generation of outliers. The remainder of the paper is organized as follows. In Section 2, our method is detailed. Section 3 is devoted to the experimental protocol and results, and Section 4 gives conclusions and future works.

\section{One-Class Random Forests}

The new discriminative approach for OCC proposed in this paper, and named One-Class Random Forests (OCRF), is an ensemble approach based on a random forest algorithm. Let us recall that the random forest (RF) principle is one of the most successful and general purpose ensemble techniques, and has shown to be competitive with state-ofthe-art classifiers like SVM and Adaboost [7/8]. It uses randomization to produce a diverse pool of individual tree-based classifiers. In the reference RF learning algorithm, two powerful randomization processes are used: bagging and Random Feature Selection (RFS). The first principle, bagging, consists in training each individual tree on a bootstrap replica of the training set. It is typically used to create the expected diversity among the individual classifiers and is particularly effective with unstable classifiers, like tree-based classifiers, in which small changes in the training set result in large changes in predictions. The second principle, RFS, is a randomization principle specifically used in tree induction algorithms. It consists, when growing the tree, in randomly selecting at each node of the tree a subset of features from which the splitting test is chosen. RFS contributes to the reduction of the dimensionality and has been shown to significantly improve RF accuracy over bagging alone [9]10].

Our OCRF algorithm includes these two randomization principles (bagging and RFS), combined with an original outlier generation process. This latter technique is usually difficult to implement since the number of outliers to generate for having reasonably good performance is exponential with respect to the size of the feature space, and may also increase as the number of available training samples increases. This issue may be addressed by sub-sampling the training set for each component classifier of the ensemble, as it is done in RF with bagging and RFS. Another popular randomization principle, the Random Subspace Method (RSM) [11], may also contribute to solve the dimensionality issue for outlier generation. It consists in randomly selecting a different subset of features for the training of each individual classifier. These two latter principles, RFS and RSM, are thus used in our method to generate outliers in smaller feature spaces. 
Now let us describe our outlier generation process. The first naive approach would be to generate outliers uniformly, before the induction of the RF. But, as mentioned above, such a process is difficult to use because of computational costs with quite large datasets, and in addition would not allow to take full advantage from ensemble methods. We thus propose to generate outliers in each bootstrap sample before the induction of each individual tree, as shown in Figure 1 It allows to reduce the number of outliers to generate, thanks to RFS and RSM that reduces the dimensionality upstream. Then, regarding the distribution of outliers, our idea is to identify areas where the target data are sparsely located in the original feature space, and to generate a lot of outliers in these areas. Conversely, fewer outliers are generated in areas containing a lot of target samples. The distribution of outliers is designed to be complementary to the distribution of targets.

The OCRF algorithm is thus made of two main steps: (i) extraction of prior information from the target data in the original feature space, in order to guide the learning process, and (ii) induction of a random forest using both RSM, that notably reduces the dimension of the feature space, and so the number of outliers to generate, and, bagging and RFS that create diversity in the pool of tree classifiers (see Figure 1). Algorithm 1 presents the detailed training algorithm of OCRF.

In summary, the OCRF method takes advantage of: (i) combining a diverse ensemble of weak and unstable classifiers, which is known to be accurate and to increase the generalization performance over single classifiers, and (ii) sub-sampling the training dataset, in terms of training samples and features, in order to efficiently generate outliers by controlling their location and their number.

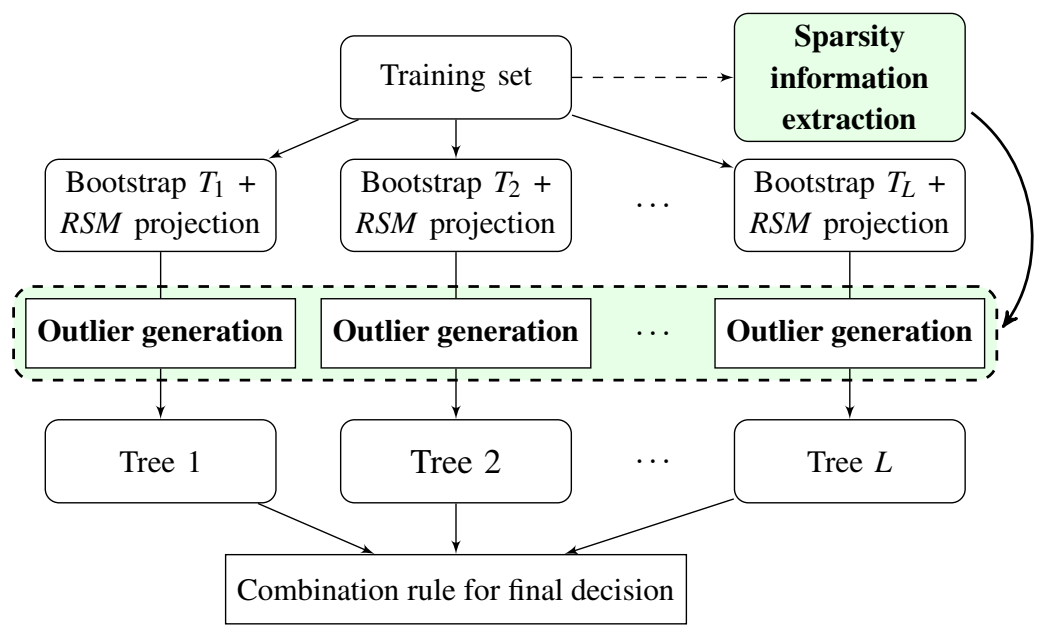

Fig. 1. Overview of the OCRF induction. Additional procedures, in comparison to a traditional $\mathrm{RF}$, are highlighted (in green and boldface). 




\section{Experiments}

In this section, we propose to experimentally assess the performance of OCRF on several public datasets and compare our approach with a few state-of-the-art one-class methods. In the following, we present the public datasets, the evaluation metrics, the one-class methods used in our comparison, and the parameters fixed for this experiment.

Datasets. Genuine one-class datasets are rare as outliers may be difficult or even impossible to sample. For testing OCC methods, authors generally transform multi-class problems into several binary classification tasks "target versus outlier", for each class of the dataset. Some authors select one class as target and label the remaining classes as outliers [12]; other authors do exactly the opposite, i.e. select one class as outlier and consider the remaining classes as the target class [12]. Thus, elaborating fair comparisons with other works based on such datasets is difficult as no clear consensus exists. We will use in our experiment the first approach that is more frequent in the literature, with one class as target and the others as outliers. We tackled in this experiment several problems of the literature, taken from 14 datasets of the recognized UC Irvine Machine Learning public repository (see Table 10. We have selected these datasets as they are often used for OCC comparison.

Evaluation Criteria. In our experiments, results are presented in terms of accuracy, but also in terms of target and outlier recognition rates, in order to allow for an analysis 
Table 1. Description of the datasets taken from the UC Irvine repository [13]

\begin{tabular}{|l|c|c|c|}
\hline & \multicolumn{3}{|c|}{ Number of } \\
\hline Dataset & attributes & classes & instances \\
\hline Sonar & 60 & 2 & 208 \\
\hline Ionosphere & 34 & 2 & 351 \\
\hline OptDigits & 64 & 10 & 5620 \\
\hline Iris & 4 & 3 & 150 \\
\hline Breast Cancer W. (bcw) & 9 & 2 & 699 \\
\hline PenDigits & 16 & 10 & 10994 \\
\hline Diabetes & 8 & 2 & 768 \\
\hline Mfeat-factors & 216 & 10 & 2000 \\
\hline Total number of one-class datasets & & $\mathbf{4 1}$ & \\
\hline
\end{tabular}

of the "target vs outlier performance" trade-off. However, these evaluation measures, widely used in the binary classification literature, do not take into account the imbalanced nature of OCC datasets [14]. Since, there is still no consensus for the performance assessment of OCC algorithms we have also used in our experiments the Matthews correlation coefficient (MCC) or "phi coefficient". It is particularly well-adapted to imbalanced problems since it takes into account the disparities in the data [14]. The MCC is based on the contingency table from the confusion matrix and is given by:

$$
\mathrm{MCC}=\frac{T P \times T N-F P \times F N}{\sqrt{(T P+F P)(T P+F N)(T N+F P)(T N+F N)}}
$$

where TP, TN, FN and FP respectively stands for true positive, true negative, false negative (or non-detection) and false positive (or false alarm). MCC values range from -1 if all predictions are wrong to +1 for perfect classification, zero values indicate that one of the two classes has not been correctly classified at all (the classifier predicts only one of the two classes).

A standard 10-fold stratified cross-validation has been repeated 5 times. The classifier performance are then averaged over the different runs.

State-of-the-Art OCC Methods and Parameterization. The OCRF algorithm is compared to four state-of-the-art OCC algorithms: the one-class SVM (OCSVM) [2] taken from the LibSVM toolbox and three density estimators, Gaussian estimator (Gauss), Parzen windows (Parzen) and Mixture of Gaussians models (MoG) taken from the Pattern Recognition Toolbox (PRTools) [15]. Each algorithm is run with the default parameterization of its toolbox 1 . Note that the definition of the threshold on the density estimator output is defined thanks to the parameter fracre $j=0.05$ of PRTools. This parameter corresponds to the fraction of legitimate target cases that will be considered as outliers during training. OCRF is also run with standard values for the parameters [10 16$]$ :

- the number of trees in the random forest is $L=200$, a value commonly considered as sufficient in practice to ensure statistical convergence of the algorithm;

${ }^{1}$ Except for the $v$ coefficient of the OCSVM, a lower bound on the fraction of support vectors, which is set to a more frequently cited value $v=0.1$, instead of $v=0.5$. 
Table 2. OCC results with MCC (accuracy is indicated in \% in brackets)

\begin{tabular}{|c|c|c|c|c|c|}
\hline Dataset & OCRF & OCSVM & Gauss & Parzen & MoG \\
\hline rsicolour & $0,579(81,5)$ & $0,897(95,3)$ & $\mathbf{0 , 9 0 3}(95,6)$ & $0,685(85,6)$ & $0,607(82,9)$ \\
\hline ginica & $0,614(82,7)$ & $\mathbf{0 , 9 0 0}(95,5)$ & $0,813(90,9)$ & $0,716(87,3)$ & $0,604(82,5)$ \\
\hline iris_setosa & $0,722(87,1)$ & $0,903(95,6)$ & $\mathbf{0 , 9 2 1}(96,4)$ & $0,799(90,9)$ & $0,643(83,3)$ \\
\hline bcw_benign & $\mathbf{0 , 9 1 9}(96,2)$ & $0,848(92,1)$ & $0,902(95,3)$ & $0,709(83,2)$ & $0,867(93,3)$ \\
\hline bcw_malignant & $\mathbf{0 , 6 2 9}(81,3)$ & $0,208(68,2)$ & $0,179(46,3)$ & $0,273(69,1)$ & $0,084(49,6)$ \\
\hline ionosphere_good & $0,683(83,3)$ & $\mathbf{0 , 7 8 5}(89,5)$ & $0,781(89,3)$ & $0,180(40,8)$ & $0,584(75,4)$ \\
\hline ionosphere_bad & $0,169(56,7)$ & $-0,348(28,2)$ & $-0,410(26,0)$ & $0,106(64,7)$ & $-0,346(33,2)$ \\
\hline Sona & $0,048(53,3)$ & $\mathbf{0 , 8 8 2}(93,6)$ & $0,342(65,9)$ & $0(46,2)$ & $0,222(47,8)$ \\
\hline ocks & $0,179(59,0)$ & $\mathbf{0 , 8 8 9}(94,0)$ & $0,120(56,3)$ & $0(53,8)$ & $0,274(56,1)$ \\
\hline S_positive & $0,139(46,4)$ & $0(65,2)$ & $0,147(35,2)$ & 0,188 & $\mathbf{0 , 2 1 9}(39,2)$ \\
\hline negative & $\mathbf{0 , 2 4 1}(68,7)$ & $0(34,8)$ & $-0,046(66,5)$ & $0,064(53,9)$ & $0,020(68,3)$ \\
\hline penc & $\mathbf{0 , 9 7 6}(99,6)$ & $0(89,6)$ & 0,970 & 0,100 & $0,961(99,3)$ \\
\hline pens & 0,585 & ,6) & 0,65 & $0,1)$ & 0,835 \\
\hline pend & $0,835(96,3)$ & $0(89,6)$ & $\mathbf{0 , 9 5 7}(99,2)$ & $0(89,6)$ & $0,956(99,2)$ \\
\hline pend & $0,918(98,5)$ & $0(90,4)$ & $\mathbf{0 , 9 6 9}(99,5)$ & $0,092(90,4)$ & $0,949(99,1)$ \\
\hline pend & 0,961 & $9,6)$ & $\mathbf{0 , 9 6 9}(99,4)$ & $0(89,6)$ & $0,953(99,1)$ \\
\hline pend & 0,756 & $0(90,4)$ & $0,880(97,8)$ & $0,092(90,4)$ & $\mathbf{0 , 9 4 2}(99,0)$ \\
\hline pend & $\mathbf{0 , 9 8 5}(99,7)$ & $0(90,4)$ & $(99,5)$ & $0(90,4)$ & $0,954(99,2)$ \\
\hline penc & 0,887 & ,6) & $7,7)$ & 0 & 0,937 \\
\hline pend & 0,634 & $0(90,4)$ & $3,2)$ & $0(90,4)$ & $\mathbf{0 , 9 5 1}(99,2)$ \\
\hline pend & $0,577(85,9)$ & $0(90,4)$ & $0,577(86,9)$ & $0,093(90,4)$ & $\mathbf{0 , 9 3 6}(98,9)$ \\
\hline opt & 0,776 & $5(90,5)$ & 0,95 & 0 & 0,745 \\
\hline optd & $0,147(26,2)$ & $0,054(89,9)$ & $\mathbf{0 , 9 3 7}$ & $0(89,8)$ & $0,803(96,7)$ \\
\hline optc & 0,143 & $0,1)$ & $9,2)$ & & $0,755(96,0)$ \\
\hline optd & $0,121(21,7)$ & $9,8)$ & $\mathbf{0 , 9 1 4}(98,4)$ & $0(89,8)$ & $0,727(95,5)$ \\
\hline optdi & 0,077 & $\overline{9,9)}$ & 0,905 & $0(89,9)$ & $0,766(96,1)$ \\
\hline optd & 0,041 & $0(90,1)$ & $\mathbf{0 , 9 5 4}(99,2)$ & $0(90,1)$ & $0,738(95,8)$ \\
\hline optd & $0,410(70,3)$ & $0,026(90,1)$ & $\mathbf{0 , 9 5 6}(99,2)$ & $0(90,1)$ & $0,778(96,3)$ \\
\hline optd & $0,264(48,2)$ & $0(89,9)$ & $\mathbf{0 , 9 3 3}(98,8)$ & $0(89,9)$ & $0,777(96,3)$ \\
\hline optd & $0,043(11,7)$ & $0(90,1)$ & $\mathbf{0 , 7 1 9}(93,6)$ & $0(90,1)$ & $0,696(95,2)$ \\
\hline optd & $0,077(15,2)$ & $0(90,0)$ & $\mathbf{0 , 8 6 0}(97,4)$ & $0(90,0)$ & $0,739(95,7)$ \\
\hline mfe & $\mathbf{0 , 8 4 4}(97,2)$ & $0,0)$ & 0,737 & $0(10,0)$ & $0(10,0)$ \\
\hline mfeat_factors_1 & $\mathbf{0 , 8 7 3}(97,8)$ & $0(90,0)$ & 0,712 & $0(10,0)$ & $0(10,0)$ \\
\hline mfeat_factors_2 & $\mathbf{0 , 8 7 9}(97,9)$ & $0(90,0)$ & $0,740(95,8)$ & $0(10,0)$ & $0(10,0)$ \\
\hline mfeat_factors_3 & $\mathbf{0 , 8 8 7}(98,0)$ & $0,017(90,0)$ & $0,695(95,1)$ & $0(10,0)$ & $0(10,0)$ \\
\hline mfeat_factors_4 & $\mathbf{0 , 8 8 4}(98,0)$ & $0(90,0)$ & $0,743(95,8)$ & $0(10,0)$ & $0(10,0)$ \\
\hline mfeat_factors_5 & $\mathbf{0 , 8 4 3}(97,3)$ & $0,013(90,0)$ & $0,738(95,8)$ & $0(10,0)$ & $0(10,0)$ \\
\hline mfeat_factors_6 & $\mathbf{0 , 9 1 0}(98,5)$ & $0,068(90,2)$ & $0,770(96,2)$ & $0(10,0)$ & $0(10,0)$ \\
\hline mfeat_factors_7 & $\mathbf{0 , 8 7 9}(97,9)$ & $0,017(90,0)$ & $0,841(97,3)$ & $0(10,0)$ & $0(10,0)$ \\
\hline ctors_8 & $0,613(90,6)$ & $0(90,0)$ & $\mathbf{0 , 6 4 7}(94,5)$ & $0(10,0)$ & $0(10,0)$ \\
\hline factors_9 & $\mathbf{0 , 8 6 6}(97,6)$ & $0,026(90,1)$ & $0,751(96,0)$ & $0(10,0)$ & $0(10,0)$ \\
\hline
\end{tabular}

- the number of attributes for the Random Subspace Method is empirically set to $K_{R S M}=10$ or $K=M$ if $M<10$, where $M$ is the dimension of the feature space;

- the number of attributes for the Random Feature Selection is $K_{R F S}=\sqrt{K_{R S M}}$. 
Table 3. Case study for results of OCRF on (a) Optical Digit and (b) MFeat-factors datasets

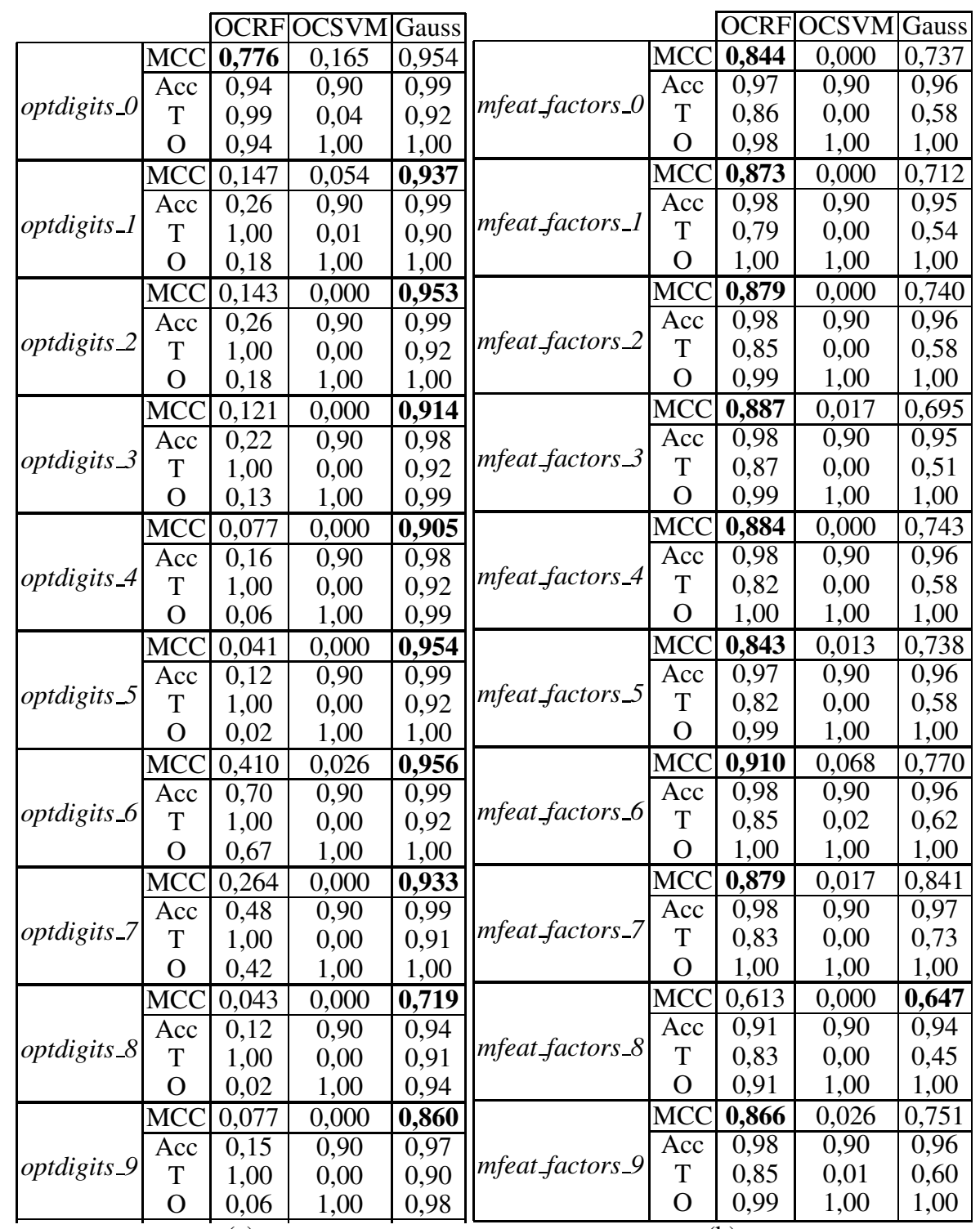

(a)

(b)

Regarding the generation of outliers during training, one must define their number and the range of their values. We have chosen the generation domain of outliers to be 1.2 times greater than the target domain estimated through the training set, assuming that the outlier domain needs to cover the whole target domain. The number of outliers to generate is empirically set to $N_{\text {outlier }}=10 \cdot N_{\text {target }}$ where $N_{\text {target }}$ is the sample size of the available training data. 
Experimental Results. The results of these experiments, in terms of accuracies and Matthews correlation coefficient (MCC) values, are presented in Table 2 In this table, the compared algorithms have MCC values of zero mostly when they always predict the outlier class except for MoG, for which MCC values of zero occur when it always predict the target class. The OCSVM classifier performs very well on Iris, bcw, Ionosphere datasets and even the best on Sonar dataset but it fails to identify outlier data on the remaining datasets.

We can observe that our method (OCRF) has no negative MCC values nor MCC values of zero. OCRF has the highest MCC values for 16 datasets among 41 while being competitive on all datasets except OptDigits datasets for which it fails to identify correctly the outlier data. The results show that all methods, except OCRF, have several negative MCC values or MCC values of zero. These methods seem to be rather unstable when dealing with some datasets: 33 datasets for OCSVM, 27 for Parzen, 11 for MoG and 2 datasets over 41 for Gauss. Gauss has an important negative value for Ionosphere $_{\text {bad }}$ dataset while it has good results in turn with Ionosphere good dataset. This latter observation often appears in Table 2, in a two-class problem, when the classifier performs well on the target class, it often fails if the second class is considered in turn as the target class.

We present in Table 3 detailed results for two handwritten digit datasets: OptDigits and MFeat-factors. For these datasets, OCRF has different results: the method performs poorly on OptDigits as it fails to identify correctly the outlier data whereas it performs the best on MFeat-factors. We can observe that, for all digit datasets, Gauss seems to be well adapted to describe each target digit cluster while OCSVM, for the same datasets, always predicts the outlier class. If we compare the MCC values to the accuracy (Acc), we can observe that MCC is more reliable to assess the performance of the method. For instance for optdigits 0 dataset, the accuracy of OCSVM is $90 \%$ while the target recognition rate is as low as $4 \%$.

\section{Conclusion and Future Works}

In this paper, we have proposed a new OCC method that is general purpose and has proved its efficiency on various public datasets. The proposed method, called One-Class Random Forest, is based on the reference random forest algorithm combined with an original procedure for generating artificial outliers. This kind of process is often used with discriminative learning methods but is difficult to implement since the number of outliers to generate for having reasonably good performance is exponential with respect to the dimension of the feature space, and may also increase as the number of available training samples increases. We have shown that the random principles used in traditional RF can be powerful tools to overcome this issue: by sub-sampling the training set for each component classifier of the ensemble, through the selection of both the training samples (with bagging) and the features (with Random Feature Selection and Random Subspace method), and by then combining all of them, we reduce the minimum number of outliers to generate and increase the generalization accuracy of the ensemble.

To assess the efficiency of our method, experiments have been conducted on several public datasets from the UCI repository and OCRF has been compared to the four most 
used OCC algorithms. On most of these datasets and using the default parameterization of each method, results have shown that OCRF performs equally well or better than these state-of-the-art OCC algorithms. Besides, OCRF appears to be rather stable on these various applications.

\section{References}

1. Hempstalk, K., Frank, E., Witten, I.: One-Class Classification by Combining Density and Class Probability Estimation. In: Daelemans, W., Goethals, B., Morik, K. (eds.) ECML PKDD 2008, Part I. LNCS (LNAI), vol. 5211, pp. 505-519. Springer, Heidelberg (2008)

2. Scholkopf, B., Platt, J., Shawe-Taylor, J., Smola, A., Williamson, R.: Estimating the support of a high-dimensional distribution. Neural Computation 13(7), 1443-1471 (2001)

3. Tarassenko, L., Clifton, D., Bannister, P., King, S., King, D.: Novelty detection. Encyclopedia of Structural Health Monitoring (2009)

4. Khan, S., Madden, M.: A survey of recent trends in one class classification. Artificial Intelligence and Cognitive Science, 188-197 (2010)

5. Tax, D., Duin, R.: Combining One-Class Classifiers. In: Kittler, J., Roli, F. (eds.) MCS 2001. LNCS, vol. 2096, pp. 299-308. Springer, Heidelberg (2001)

6. Dietterich, T.: Ensemble Methods in Machine Learning. In: Kittler, J., Roli, F. (eds.) MCS 2000. LNCS, vol. 1857, pp. 1-15. Springer, Heidelberg (2000)

7. Breiman, L.: Random forests. Machine Learning 45(1), 5-32 (2001)

8. Robnik-Sikonja, M.: Improving Random Forests. In: Boulicaut, J.-F., Esposito, F., Giannotti, F., Pedreschi, D. (eds.) ECML 2004. LNCS (LNAI), vol. 3201, pp. 359-370. Springer, Heidelberg (2004)

9. Bernard, S., Heutte, L., Adam, S.: Forest-rk: A new random forest induction method. In: Advanced Intelligent Computing Theories and Applications. With Aspects of Artificial Intelligence, pp. 430-437 (2008)

10. Geurts, P., Ernst, D., Wehenkel, L.: Extremely randomized trees. Machine Learning 63(1), 3-42 (2006)

11. Ho, T.: The random subspace method for constructing decision forests. IEEE Transactions on Pattern Analysis and Machine Intelligence 20(8), 832-844 (1998)

12. Tax, D., Ypma, A., Duin, R.: Support vector data description applied to machine vibration analysis. In: Proc. 5th Annual Conference of the Advanced School for Computing and Imaging, Heijen, NL, Citeseer (1999)

13. Blake, C., Merz, C.: Uci repository of machine learning databases. Department of Information and Computer Science, vol. 55. University of California, Irvine (1998), http://www.ics.uci.edu/ mlearn/mlrepository.html

14. Baldi, P., Brunak, S., Chauvin, Y., Andersen, C., Nielsen, H.: Assessing the accuracy of prediction algorithms for classification: an overview. Bioinformatics 16(5), 412-424 (2000)

15. Duin, R.: PRTools version 3.0: A matlab toolbox for pattern recognition. In: Proc. of SPIE, Citeseer (2000)

16. Bernard, S., Heutte, L., Adam, S.: Influence of Hyperparameters on Random Forest Accuracy. In: Benediktsson, J.A., Kittler, J., Roli, F. (eds.) MCS 2009. LNCS, vol. 5519, pp. 171-180. Springer, Heidelberg (2009) 